\title{
603.
}

\section{ON THE POTENTIAL OF THE ELLIPSE AND THE CIRCLE.}

[From the Proceedings of the London Mathematical Society, vol. VI. (1874-1875), pp. 38-58. Read January 14, 1875.]

The Potential of the Ellipse.

1. I CONSIDER the potential of an ellipse (or say an elliptic plate of uniform density); viz. this is

$$
V=\int \frac{d x d y}{\sqrt{(a-x)^{2}+(b-y)^{2}+c^{2}}}
$$

the limits being given by the equation $\frac{x^{2}}{f^{2}}+\frac{y^{2}}{g^{2}}=1$.

Writing herein $x=m f \cos u, y=m g \sin u$, we have $d x d y=f g m d m d u$; and consequently

$$
V=f g \int \frac{m d m d u}{\sqrt{(a-m f \cos u)^{2}+(b-m g \sin u)^{2}+c^{2}}},
$$

where the integrations are to be taken from $m=0$ to $m=1$, and from $u=0$ to $u=2 \pi$.

2. It is to be remarked that, by first performing the integration in regard to $m$, we may reduce the potential to the form $\int d u . F$, where $F$ is an algebraic function of $\cos u, \sin u$; and that the result so obtained, although in the general case too complex to be manageable, is a useful one in the case $f=g$, where the ellipse becomes a circle. The case of the circle will be treated of separately, but in the general case it will be sufficient to show that the integral is of the form in question.

c. IX. 
3. To accomplish this, writing

$$
\begin{aligned}
& A=a^{2}+b^{2}+c^{2} \\
& B=a f \cos u+b g \sin u \\
& C=f^{2} \cos ^{2} u+g^{2} \sin ^{2} u
\end{aligned}
$$

then the integral in regard to $m$ is

which is

$$
\int \frac{m d m}{\sqrt{A-2 B m+C m^{2}}}
$$

$$
=\frac{1}{C} \sqrt{A-2 B m+C m^{2}}+\frac{B}{C \sqrt{C}} \log \left\{C m-B+\sqrt{C} \sqrt{A-2 B m+C m^{2}}\right\} .
$$

Taken between the limits 0 and 1 , this is

$$
=\frac{1}{C}(\sqrt{A-2 B+C}-\sqrt{A})+\frac{B}{C \sqrt{C}} \log \left\{\frac{C-B+\sqrt{C} \sqrt{A-2 B+C}}{-B+\sqrt{C} \sqrt{A}}\right\} ;
$$

and we have therefore

$$
V=f g \int d u \frac{1}{C}(\sqrt{A-2 B+C}-\sqrt{A})+f g \int d u \frac{(a f \cos u+b g \sin u)}{\left(f^{2} \cos ^{2} u+g^{2} \sin ^{2} u\right)^{\frac{3}{2}}} \log \Upsilon,
$$

where, for greater clearness, the value of the coefficient $\frac{B}{C \sqrt{C}}$ of the logarithmic term has been written at full length.

4. But this coefficient admits of algebraic integration, viz. we have

$$
f g \int d u \frac{a f \cos u+b g \sin u}{\left(f^{2} \cos ^{2} u+g^{2} \sin ^{2} u\right)^{\frac{3}{2}}}=\frac{a g \sin u-b f \cos u}{\left(f^{2} \cos ^{2} u+g^{2} \sin ^{2} u\right)^{\frac{1}{2}}}
$$

hence, integrating the second term by parts, we have

$$
\begin{aligned}
V= & f g \int d u \frac{1}{C}\{\sqrt{A-2 B+C}-\sqrt{A}\} \\
& +\frac{a g \sin u-b f \cos u}{\left(f^{2} \cos ^{2} u+g^{2} \sin ^{2} u\right)^{\frac{1}{2}}} \log \Upsilon \\
& -\int d u \frac{a g \sin u-b f \cos u}{\left(f^{2} \cos ^{2} u+g^{2} \sin ^{2} u\right)^{\frac{1}{2}}} \cdot \frac{\Upsilon^{\prime}}{\Upsilon}
\end{aligned}
$$

where the second term, taken between the limits $u=0, u=2 \pi$, is $=0$; and $\frac{\Upsilon^{\prime}}{\Upsilon}$ being an algebraic function of $\sin u, \cos u$, the potential is expressed in the form in question.

5. But we may, by means of a transformation upon $u$ (that made use of in Gauss' Memoir* on the attraction of an elliptic ring), transform the expression so as

* [Ges. Werke, t. III., pp. 333-355; in particular, l.c., p. 338]. 
to obtain the integral in regard to $m$ under a much more simple form. We, in fact, assume

$$
\begin{aligned}
& \cos u=\frac{\alpha+\alpha^{\prime} \cos T+\alpha^{\prime \prime} \sin T}{\gamma+\gamma^{\prime} \cos T+\gamma^{\prime \prime} \sin T}, \\
& \sin u=\frac{\beta+\beta^{\prime} \cos T+\beta^{\prime \prime} \sin T}{\gamma+\gamma^{\prime} \cos T+\gamma^{\prime \prime} \sin T},
\end{aligned}
$$

where the nine coefficients are such that identically

$\left(\alpha+\alpha^{\prime} \cos T+\alpha^{\prime \prime} \sin T\right)^{2}+\left(\beta+\beta^{\prime} \cos T+\beta^{\prime \prime} \sin T\right)^{2}-\left(\gamma+\gamma^{\prime} \cos T+\gamma^{\prime \prime} \sin T\right)^{2}=\cos ^{2} T+\sin ^{2} T-1$,

(this of course renders the two equations consistent); and also that

$$
(a-m f \cos u)^{2}+(b-m g \sin u)^{2}+c^{2}=\frac{1}{\left(\gamma+\gamma^{\prime} \cos T+\gamma^{\prime \prime} \sin T\right)^{2}}\left(G+G^{\prime} \cos ^{2} T+G^{\prime \prime} \sin ^{2} T\right) .
$$

This last condition gives, for the determination of the coefficients $G, G^{\prime}, G^{\prime \prime}$, the identity

$$
(\theta-G)\left(\theta+G^{\prime}\right)\left(\theta+G^{\prime \prime}\right)=-\left(\theta+m^{2} f^{2}\right)\left(\theta+m^{2} g^{2}\right) \theta\left\{\frac{a^{2}}{\theta+m^{2} f^{2}}+\frac{b^{2}}{\theta+m^{2} g^{2}}+\frac{c^{2}}{\theta}-1\right\}
$$

or, what is the same thing, $G,-G^{\prime},-G^{\prime \prime}$ are the roots of the equation

$$
\frac{a^{2}}{\theta+m^{2} f^{2}}+\frac{b^{2}}{\theta+m^{2} g^{2}}+\frac{c^{2}}{\theta}-1=0 .
$$

This equation has one positive root, which may be taken to be $G$, and two negative roots, which will then be $-G^{\prime},-G^{\prime \prime}$; viz. $G, G^{\prime}, G^{\prime \prime}$ are thus all positive; and $G$ denotes the positive root of the last-mentioned equation.

6. We have

and thence

$$
d u=\frac{d T}{\left(G+G^{\prime} \cos T+G^{\prime \prime} \sin T\right)^{2}}
$$

$$
V=f g \int m d m \int \frac{d T}{\left(G+G^{\prime} \cos ^{2} T+G^{\prime \prime} \sin ^{2} T\right)^{\frac{1}{2}}},
$$

the integral in regard to $T$ being taken from 0 to $2 \pi$; or, what is the same thing, we may multiply by 4 and take the integral only from 0 to $\frac{\pi}{2}$; viz. we thus have

$$
V=4 f g \int m d m \int_{0}^{\frac{1}{2} \pi} \frac{d T}{\left(G+G^{\prime} \cos ^{2} T+G^{\prime \prime} \sin ^{2} T\right)^{\frac{1}{2}}},
$$

where the integral in regard to $T$ can be at once reduced to the standard form of an elliptic function, or it might be calculated by Gauss' method of the arithmeticogeometrical mean.

7. But, for the present purpose, a further reduction is required. Writing

$$
t=G+\left(G+G^{\prime}\right) \cot ^{2} T
$$


we have

$$
\begin{aligned}
& t-G=\left(G+G^{\prime}\right) \frac{\cos ^{2} T}{\sin ^{2} T} \\
& t+G^{\prime}=\left(G+G^{\prime}\right) \frac{1}{\sin ^{2} T} \\
& t+G^{\prime \prime}=\left(G+G^{\prime} \cos ^{2} T+G^{\prime \prime} \sin ^{2} T\right) \frac{1}{\sin ^{2} T}
\end{aligned}
$$

whence

$$
\sqrt{ } t-G \cdot t+G^{\prime} \cdot t+G^{\prime \prime}=\left(G+G^{\prime}\right)\left(G+G^{\prime} \cos ^{2} T+G^{\prime \prime} \sin ^{2} T\right)^{\frac{1}{2}} \frac{\cos T}{\sin ^{3} T}
$$

moreover

$$
d t=-2\left(G+G^{\prime}\right) \frac{\cos T}{\sin ^{3} T} d T
$$

Hence

$$
\frac{d t}{\sqrt{t-G \cdot t+G^{\prime} \cdot t+G^{\prime \prime}}}=\frac{-2 d T}{\left\{G+G^{\prime} \cos ^{2} T+G^{\prime \prime} \sin ^{2} T\right\}^{\frac{1}{2}}}
$$

and, observing that to the limits $0, \frac{\pi}{2}$ of $T$ correspond the limits $\infty, G$ of $t$, we thence obtain

$$
V=2 f g \int m d m \int_{G}^{\infty} \frac{d t}{\sqrt{t-G \cdot t+G^{\prime} \cdot t+G^{\prime \prime}}}
$$

or, what is the same thing,

$$
V=2 f g \int m d m \int_{G}^{\infty} \frac{d t}{\sqrt{t\left(t+m^{2} f^{2}\right)\left(t+m^{2} g^{2}\right)\left(1-\frac{a^{2}}{t+m^{2} f^{2}}-\frac{b^{2}}{t+m^{2} g^{2}}-\frac{c^{2}}{t}\right)}}
$$

where $G$ denotes, as before, the positive root of the equation

$$
\frac{a^{2}}{\theta+m^{2} f^{2}}+\frac{b^{2}}{\theta+m^{2} g^{2}}+\frac{c^{2}}{\theta}-1=0
$$

8. Writing for $t, m^{2} t$, and for $G, m^{2} G$, the formula becomes

$$
V=2 f g \int m d m \int_{G}^{\infty} \frac{d t}{\sqrt{t \cdot t+f^{2} \cdot t+g^{2}\left(m^{2}-\frac{a^{2}}{t+f^{2}}-\frac{b^{2}}{t+g^{2}}-\frac{c^{2}}{t}\right)}},
$$

where $G$ now denotes the positive root of the equation

$$
\frac{a^{2}}{\theta+f^{2}}+\frac{b^{2}}{\theta+g^{2}}+\frac{c^{2}}{\theta}-m^{2}=0
$$


Thus $G$ is a function of $m$; but it is to be remarked that the integration in respect to $m$ can be performed through the integral sign $\int_{G}^{\infty} d t$ in precisely the same way as if $G$ were constant, and that we, in fact, have

$$
V=2 f g\left[\int_{G}^{\infty} d t \sqrt{m^{2}-\frac{a^{2}}{t+f^{2}}-\frac{b^{2}}{t+g^{2}}-\frac{c^{2}}{t}} \frac{1}{\sqrt{t \cdot t+f^{2} \cdot t+g^{2}}}\right],
$$

where the function of $m$ is to be taken between the limits 0 and 1 . The reason is that, differentiating this last integral in respect to $m$, the term depending on the variation of the limit $G$ is

$$
-\sqrt{m^{2}-\frac{a^{2}}{G+f^{2}}-\frac{b^{2}}{G+g^{2}}-\frac{c^{2}}{G}} \frac{1}{\sqrt{G \cdot G+f^{2} \cdot G+g^{2}}} \frac{d G}{d m},
$$

which is $=0$ in virtue of the equation which defines $G$; hence the whole result is the term arising from the variation of $m$ in so far as it appears explicitly.

9. Proceeding next to take the function of $m$ between the two limits: for $m=0$ we have $G=\infty$, and the integral vanishes; for $m=1$ we have $G$ the positive root of the equation

$$
\frac{a^{2}}{\theta+f^{2}}+\frac{b^{2}}{\theta+g^{2}}+\frac{c^{2}}{\theta}-1=0,
$$

or, using $\theta$ to denote the positive root of this equation, the value is $G=\theta$; we thus finally obtain

$$
V=2 f g \int_{\theta}^{\infty} d t \sqrt{1-\frac{a^{2}}{t+f^{2}}-\frac{b^{2}}{t+g^{2}}-\frac{c^{2}}{t}} \frac{1}{\sqrt{t \cdot t+f^{2} \cdot t+g^{2}}}
$$

as the expression for the potential of the ellipse semiaxes $(f, g)$ on the point $(a, b, c)$.

\section{Case where the Attracted Point is on the Focal Hyperbola.}

10. The result becomes very simple when the attracted point is in the focal hyperbola of the ellipse, viz. when we have $b=0$ and $\frac{a^{2}}{f^{2}-g^{2}}-\frac{c^{2}}{g^{2}}=1$. The function $1-\frac{a^{2}}{t+f^{2}}-\frac{b^{2}}{t+g^{2}}-\frac{c^{2}}{t}$ is here

$$
\begin{aligned}
& =\frac{a^{2}}{f^{2}-g^{2}}-\frac{c^{2}}{g^{2}}-\frac{a^{2}}{f^{2}+t}-\frac{c^{2}}{t} \\
& =\left(t+g^{2}\right)\left\{\frac{a^{2}}{\left(t+f^{2}\right)\left(f^{2}-g^{2}\right)}-\frac{c^{2}}{g^{2} t}\right\} \\
& =\left(t+g^{2}\right)\left\{\left(1+\frac{c^{2}}{g^{2}}\right) \frac{1}{t+f^{2}}-\frac{c^{2}}{g^{2} t}\right\} \\
& =\frac{t+g^{2}}{t\left(t+f^{2}\right)}\left(t-\frac{c^{2} f^{2}}{g^{2}}\right) .
\end{aligned}
$$


Hence also $\theta=\frac{c^{2} f^{2}}{g^{2}} ;$ introducing this value, the function in question becomes

and we have

$$
=\frac{\left(t+g^{2}\right)(t-\theta)}{t\left(t+f^{2}\right)}
$$

$$
\begin{aligned}
V & =2 f g \int_{\theta}^{\infty} d t \frac{\sqrt{t+g^{2} \cdot t-\theta}}{\sqrt{t \cdot t+f^{2}}} \frac{1}{\sqrt{t \cdot t+f^{2} \cdot t+g^{2}}} \\
& =2 f g \int_{\theta}^{\infty} \frac{d t \sqrt{t-\theta}}{t \cdot t+f^{2}}
\end{aligned}
$$

which, writing $t=x^{2}+\theta$, becomes

$$
\begin{aligned}
V & =4 f g \int_{0}^{\infty} \frac{x^{2} d x}{x^{2}+\theta \cdot x^{2}+\theta+f^{2}} \\
& =\frac{4 f g}{f^{2}} \int_{0}^{\infty}\left(\frac{\theta+f^{2}}{x^{2}+\theta+f^{2}}-\frac{\theta}{x^{2}+\theta}\right) d x \\
& =\frac{4 g}{f}\left(\sqrt{\theta+f^{2}} \tan ^{-1} \frac{x}{\sqrt{\theta+f^{2}}}-\sqrt{ } \theta \tan ^{-1} \frac{x}{\sqrt{ } \theta}\right)_{0}^{\infty} \\
& =2 \pi \frac{g}{f}\left(\sqrt{\theta+f^{2}}-\sqrt{ } \theta\right)
\end{aligned}
$$

or, substituting for $\theta$ its value $\frac{c^{2} f^{2}}{g^{2}}$, this is

$$
V=2 \pi\left(\sqrt{c^{2}+g^{2}}-c\right),
$$

which is, in fact, the potential of the circle $x^{2}+y^{2}=g^{2}$ on the axial point $(0,0, c)$; and, observing that the value is independent of $f$, we have at once the theorem that, considering $f$ as variable, and taking the attracted point at the constant altitude $c$ in the focal hyperbola $\frac{x^{2}}{f^{2}-g^{2}}-\frac{z^{2}}{g^{2}}=1$, the potential $i_{\text {w }}$ the same, whatever is the value of the semi-axis major $f$ of the ellipse.

11. A point in the focal hyperbola determines, with the ellipse, a right circular cone having for its axis the tangent to the hyperbola; viz. the tangent in question is equally inclined to the two lines joining the point with the foci of the hyperbola, or with the extremities of the major axis of the ellipse. Taking $\theta$ for the inclination of the tangent to either of these lines, viz. $\theta$ is the semi-aperture of the cone, and $\gamma$ for the inclination of the tangent to the axis of $z$, then it is easy to show that

and we thence have

$$
\sqrt{c^{2}+g^{2}}=c \frac{\cos \gamma}{\sqrt{\cos ^{2} \gamma-\sin ^{2} \theta}}
$$

$$
V=2 \pi c\left(\frac{\cos \gamma}{\sqrt{\cos ^{2} \gamma-\sin ^{2} \theta}}-1\right)
$$


viz. the ellipse is here considered as the section of a right cone of semi-aperture $\theta$, the perpendicular distance from the vertex being $=c$, and the inclination of this distance to the axis of the cone being $=\gamma$; and this being so, the potential is then expressed by the last preceding equation. It will be observed that, when $\gamma=\frac{\pi}{2}-\theta$, the section becomes a parabola, and the potential is infinite; for any larger value of $\gamma$, the section is a hyperbola, and the formula ceases to be applicable.

12. I originally obtained the result by thus considering the ellipse as the section of a right cone. Consider for a moment, in the case of any cone whatever, the plate included between the plane, perpendicular distance from the vertex $=c$, and the consecutive parallel plane, distance $=c+d c$. Let $d \Sigma$ denote an element of the first plane, $r$ its distance from the vertex, and $r+d r$ the distance produced to meet the second plane; also let $d \omega$ denote the subtended solid angle. We have $d \Sigma d c=r^{2} d r d \omega$, or, since $\frac{d c}{c}=\frac{d r}{r}$, we obtain $d \Sigma=\frac{1}{c} r^{3} d \omega$, or $\frac{1}{r} d \Sigma=\frac{1}{c} r^{2} d \omega$; wherefore the potential of the plane section is $V=\frac{1}{c} \int r^{2} d \omega$, where $r$ denotes the value at a point of the plane section, and the integration extends over the spherical aperture of the cone.

13. Let the position of $r$ be determined by means of its inclination $\theta$ to the axis of the cone, and the azimuth $\phi$ of the plane through $r$ and the axis of the cone; viz. taking the axis of the cone for the axis of $z$, suppose, as usual, $x=r \sin \theta \cos \phi$, $y=r \sin \theta \sin \phi, \quad z=r \cos \theta$. We have then, as usual, $d \omega=\sin \theta d \theta d \phi$; and if the equation of the plane be $x \cos \alpha+y \cos \beta+z \cos \gamma=c$, then the value of $r$ is obtained from the equation

$$
r\{(\cos \alpha \cos \phi+\cos \beta \sin \phi) \sin \theta+\cos \gamma \cos \theta\}=c ;
$$

so that we have for the potential

$$
V=c \int \frac{\sin \theta d \theta d \phi}{\{(\cos \alpha \cos \phi+\cos \beta \sin \phi) \sin \theta+\cos \gamma \cos \theta\}^{2}},
$$

where the integration is extended over the whole spherical aperture of the cone; viz. in the case of a right cone of semi-aperture $\theta$, the limits are from $\theta=0$ to $\theta=\theta$ and from $\phi=0$ to $\phi=2 \pi$.

14. Write

$$
(\cos \alpha \cos \phi+\cos \beta \sin \phi) \sin \theta+\cos \gamma \cos \theta=M \cos (\theta-N),
$$

where $M, N$ are given functions of $\phi$; then we have

and the $\theta$-integral is

$$
V=c \int \frac{d \phi}{M^{2}} \int \frac{\sin \theta d \theta}{\cos ^{2}(\theta-N)}
$$

$$
\begin{gathered}
\int \frac{[\sin (\theta-N) \cos N+\cos (\theta-N)}{\cos ^{2}(\theta-N)} \frac{\sin N] d \theta}{=}, \\
=\cos N \sec (\theta-N)+\sin N \log \tan \left\{\frac{1}{4} \pi+\frac{1}{2}(\theta-N)\right\},
\end{gathered}
$$


which between the limits is

$$
=\cos N\{\sec (\theta-N)-\sec N\}+\sin N\left\{\log \tan \left[\frac{1}{4} \pi+\frac{1}{2}(\theta-N)\right]-\log \tan \left(\frac{1}{4} \pi-\frac{1}{2} N\right)\right\},
$$

$\theta$ now denoting the semi-aperture of the right cone. And we have

$$
V=c \int \frac{d \phi}{M^{2}}\left\{\cos N\left(\frac{1}{\cos (N-\theta)}-\frac{1}{\cos N}\right)+\sin N\left[\log \tan \left\{\frac{1}{4} \pi+\frac{1}{2}(\theta-N)\right\}-\log \tan \left(\frac{1}{4} \pi-\frac{1}{2} N\right)\right]\right\} .
$$

We may without loss of generality write $\cos \beta=0$, and therefore $\cos \alpha=\sin \gamma$, where $\gamma$ now is the inclination of the perpendicular on the plane to the axis of the cone. We thus have

that is,

$$
\cos \gamma \cos \theta+\sin \gamma \cos \phi \sin \theta=M \cos (\theta-N),
$$

$$
\begin{aligned}
\cos \gamma & =M \cos N, \\
\sin \gamma \cos \phi & =M \sin N ;
\end{aligned}
$$

whence

$$
\begin{aligned}
\tan N & =\tan \gamma \cos \phi \text { or } N=\tan ^{-1}(\tan \gamma \cos \phi), \\
M^{2} & =\cos ^{2} \gamma+\sin ^{2} \gamma \cos ^{2} \phi=1-\sin ^{2} \gamma \sin ^{2} \phi,
\end{aligned}
$$

and

$$
\frac{\cos N}{\cos (N-\theta)}=\frac{1}{\cos \theta+\sin \theta \tan \gamma \cos \phi} \text {. }
$$

15. We have, therefore,

But

$$
\begin{array}{r}
V=c \int \frac{d \phi}{1-\sin ^{2} \gamma \sin ^{2} \phi}\left(\frac{1}{\cos \theta+\sin \theta \tan \gamma \cos \phi}-1\right) \\
+c \int \frac{d \phi \sin \gamma \cos \phi}{\left(1-\sin ^{2} \gamma \sin ^{2} \phi\right)^{\frac{1}{2}}}\left\{\log \tan \left[\frac{1}{4} \pi+\frac{1}{2} \theta-\frac{1}{2} \tan ^{-1}(\tan \gamma \cos \phi)\right]\right. \\
\left.-\log \tan \left[\frac{1}{4} \pi-\frac{1}{2} \tan ^{-1}(\tan \gamma \cos \phi)\right]\right\} .
\end{array}
$$

$$
\int \frac{d \phi \cos \phi}{\left(1-\sin ^{2} \gamma \sin ^{2} \phi\right)^{\frac{3}{2}}}=\frac{\sin \phi}{\left(1-\sin ^{2} \gamma \sin ^{2} \phi\right)^{\frac{1}{2}}}
$$

hence the second line is

$$
\begin{aligned}
& c \sin \gamma \frac{\sin \phi}{\left(1-\sin ^{2} \gamma \sin ^{2} \phi\right)^{\frac{1}{2}}}\left\{\operatorname { l o g } \operatorname { t a n } \left[\frac{1}{4} \pi\right.\right.\left.+\frac{1}{2} \theta-\frac{1}{2} \tan ^{-1}(\tan \gamma \cos \phi)\right] \\
&\left.-\log \tan \left[\frac{1}{4} \pi-\frac{1}{2} \tan ^{-1}(\tan \gamma \cos \phi)\right]\right\} \\
&-c \sin \gamma \int d \phi \frac{\sin \phi}{\left(1-\sin ^{2} \gamma \sin ^{2} \phi\right)^{\frac{1}{2}}} \frac{d}{d \phi}\left\{\log \tan \left[\frac{1}{4} \pi+\frac{1}{2} \theta-\frac{1}{2} \tan ^{-1}(\tan \gamma \cos \phi)\right]\right. \\
&\left.-\log \tan \left[\frac{1}{4} \pi-\frac{1}{2} \tan ^{-1}(\tan \gamma \cos \phi)\right]\right\} .
\end{aligned}
$$

But, restoring for a moment $N$ in place of $\tan ^{-1}(\tan \gamma \cos \phi)$, we have

$$
\begin{aligned}
& \frac{d}{d \phi} \log \tan \left(\frac{1}{4} \pi+\frac{1}{2} \theta-N\right)=-\frac{d N}{d \phi} \frac{1}{\cos (N-\theta)}=\frac{\sin \gamma \cos \gamma \sin \phi}{1-\sin ^{2} \gamma \sin ^{2} \phi} \frac{1}{\cos (N-\theta)} \\
& \frac{d}{d \phi} \log \tan \left(\frac{1}{4} \pi-N\right) \quad=-\frac{d N}{d \phi} \frac{1}{\cos N}=\frac{\sin \gamma \cos \gamma \sin \phi}{1-\sin ^{2} \gamma \sin ^{2} \phi} \frac{1}{\cos N}
\end{aligned}
$$


And then, in place of $\frac{1}{\cos (N-\theta)}-\frac{1}{\cos N}$, writing

$$
\frac{1}{\cos \gamma \sqrt{1-\sin ^{2} \gamma \sin ^{2} \phi}}\left(\frac{1}{\cos \theta+\sin \theta \tan \gamma \cos \phi}-1\right)
$$

the expression in question becomes

$$
\begin{gathered}
c \sin \gamma \frac{\sin \phi}{\left(1-\sin ^{2} \gamma \sin ^{2} \phi\right)^{\frac{1}{2}}}\left\{\log \tan \left[\frac{1}{4} \pi+\frac{1}{2} \theta-\frac{1}{2} \tan ^{-1}(\tan \gamma \cos \phi)\right]\right. \\
\left.-\log \tan \left[\frac{1}{4} \pi-\frac{1}{2} \tan ^{-1}(\tan \gamma \cos \phi)\right]\right\} \\
-c \int d \phi \frac{\sin ^{2} \gamma \sin ^{2} \phi}{1-\sin ^{2} \gamma \sin ^{2} \phi}\left(\frac{1}{\cos \theta+\sin \theta \tan \gamma \cos \phi}-1\right) .
\end{gathered}
$$

And we have

$$
\begin{aligned}
& V=\frac{c \sin \gamma \sin \phi}{\left(1-\sin ^{2} \gamma \sin ^{2} \phi\right)^{\frac{1}{2}}}\left\{\log \tan \left[\frac{1}{4} \pi+\frac{1}{2} \theta-\frac{1}{2} \tan ^{-1}(\tan \gamma \cos \phi)\right]\right. \\
& \left.\quad-\log \tan \left[\frac{1}{4} \pi-\frac{1}{2} \tan ^{-1}(\tan \gamma \cos \phi)\right]\right\}+c \int d \phi\left(\frac{1}{\cos \theta+\sin \theta \tan \gamma \cos \phi}-1\right) .
\end{aligned}
$$

16. The integral is here

$$
\begin{aligned}
& =\int d \phi\left\{\frac{\cos \gamma(\cos \theta \cos \gamma-\sin \theta \sin \gamma \cos \phi)}{\cos ^{2} \theta \cos ^{2} \gamma-\sin ^{2} \theta \sin ^{2} \gamma \cos ^{2} \phi}-1\right\} \\
& =\cos ^{2} \gamma \cos \theta \int \frac{d \phi}{\cos ^{2} \theta \cos ^{2} \gamma-\sin ^{2} \theta \sin ^{2} \gamma \cos ^{2} \phi} \\
& \quad-\cos \gamma \sin \gamma \sin \theta \int \frac{\cos \phi d \phi}{\cos ^{2} \theta \cos ^{2} \gamma-\sin ^{2} \theta \sin ^{2} \gamma \cos ^{2} \phi}-\int d \phi \\
& =\frac{\cos \gamma}{\sqrt{\cos ^{2} \gamma-\sin ^{2} \theta}} \tan ^{-1} \frac{\cos \theta \cos \gamma \tan \phi}{\sqrt{\cos ^{2} \gamma-\sin ^{2} \theta}} \\
& \quad-\frac{\cos \gamma}{\sqrt{\cos ^{2} \gamma-\sin ^{2} \theta}} \tan ^{-1} \frac{\sin \theta \sin \gamma \sin \phi}{\sqrt{\cos ^{2} \gamma-\sin ^{2} \theta}-\phi,}
\end{aligned}
$$

as may be immediately verified.

Hence

$$
\begin{aligned}
V=\frac{c \sin \gamma \sin \phi}{\sqrt{1-\sin ^{2} \gamma \sin ^{2} \phi}}\left\{\operatorname { l o g } \operatorname { t a n } \left[\frac{1}{4} \pi\right.\right. & \left.+\frac{1}{2} \theta-\frac{1}{2} \tan ^{-1}(\tan \gamma \cos \phi)\right] \\
& \left.-\log \tan \left[\frac{1}{4} \pi-\frac{1}{2} \tan ^{-1}(\tan \gamma \cos \phi)\right]\right\} \\
& +\frac{c \cos \gamma}{\sqrt{\cos ^{2} \theta-\sin ^{2} \gamma}} \tan ^{-1} \frac{\cos \theta \cos \gamma \tan \phi}{\sqrt{\cos ^{2} \theta-\sin ^{2} \gamma}} \\
& -\frac{c \cos \gamma}{\sqrt{\cos ^{2} \theta-\sin ^{2} \gamma}} \tan ^{-1} \frac{\sin \theta \sin \gamma \sin \phi}{\sqrt{\cos ^{2} \theta-\sin ^{2} \gamma}} \\
& -c \phi
\end{aligned}
$$

which is to be taken between the limits 0 and $2 \pi$; or, what is the same thing, the integral may be taken between the limits $0, \pi$, and multiplied by 2 . But as $\phi$ passes c. IX. 
from 0 to $\pi$, the arc of the form $\tan ^{-1}(A \tan \phi)$ passes through the values $0, \frac{\pi}{2},-\frac{\pi}{2}, 0$, but the other arc of the form $\tan ^{-1}(B \sin \phi)$ through the values $0, \frac{\pi}{2}, \frac{\pi}{2}, 0$; the first arc gives therefore a term $\pi$, the second arc a term 0 , and the final result is

which is right.

$$
V=2 c \pi\left(\frac{\cos \gamma}{\sqrt{\cos ^{2} \gamma-\sin ^{2} \theta}}-1\right)
$$

\section{The Potential of the Circle.}

17. In the case of the circle we have $g=f$; the terms containing $a^{2}, b^{2}$ unite throughout into a single term containing $a^{2}+b^{2}$, and there is obviously no loss of generality in assuming $b=0$, and so reducing this to $a^{2}$; viz. we take the axis of $x$ to pass through the projection of the attracted point, the coordinates of this point being therefore $(a, 0, c)$. We in fact consider the potential

$$
V=\int \frac{d x d y}{\sqrt{(a-x)^{2}+y^{2}+c^{2}}}
$$

over the circle $x^{2}+y^{2}=f^{2}$; or, writing $x=m f \cos \phi, y=m f \sin \phi$, we have $d x d y=f^{2} m d m d \phi$, and therefore

$$
V=f^{2} \int \frac{m d m d \phi}{\sqrt{a^{2}+c^{2}+m^{2} f^{2}-2 m a f \cos \phi}},
$$

the integral being taken from $m=0$ to $m=1$, and $\phi=0$ to $\phi=2 \pi$.

Writing in the general formula $g=f$ and $b=0$, we have

$$
V=2 f^{2} \int_{\theta}^{\infty} \frac{d t \sqrt{1-\frac{a^{2}}{t+f^{2}}-\frac{c^{2}}{t}}}{\left(t+f^{2}\right) \sqrt{t}}
$$

where $\theta$ denotes the positive root of the equation

or, observing that

$$
1-\frac{a^{2}}{\theta+f^{2}}-\frac{c^{2}}{\theta}=0
$$

$$
\begin{aligned}
1-\frac{a^{2}}{t+f^{2}}-\frac{c^{2}}{t} & =a^{2}\left(\frac{1}{\theta+f^{2}}-\frac{1}{t+f^{2}}\right)+c^{2}\left(\frac{1}{\theta}-\frac{1}{t}\right) \\
& =(t-\theta)\left\{\frac{a^{2}}{\left(\theta+f^{2}\right)\left(t+f^{2}\right)}+\frac{c^{2}}{\theta t}\right\} \\
& =\frac{t-\theta}{t \cdot t+f^{2}}\left\{\left(1-\frac{c^{2}}{\theta}\right) t+\frac{c^{2}}{\theta}\left(t+f^{2}\right)\right\} \\
& =\frac{(t-\theta)\left(t+\frac{c^{2} f^{2}}{\theta}\right)}{t \cdot t+f^{2}}
\end{aligned}
$$


we have also

$$
V=2 f^{2} \int_{\theta}^{\infty} \frac{\sqrt{\left(t-\theta \cdot t+\frac{c^{2} f^{2}}{\theta}\right)} d t}{t\left(t+f^{2}\right) \sqrt{t+f^{2}}}
$$

18. The present particular case gives rise to some interesting investigations. We may, in the first place, complete the process of first integrating directly in regard to $\mathrm{m}$.

Writing

$$
V=f \int\left\{\begin{array}{l}
{[(m f-a \cos \phi)+a \cos \phi] d m d \phi} \\
\left\{(m f-a \cos \phi)^{2}+a^{2} \sin ^{2} \phi+c^{2}\right\}^{\frac{1}{2}}
\end{array}\right.
$$

the integral in regard to $m$ is

$$
=\frac{1}{f}\left\{\sqrt{(m f-a \cos \phi)^{2}+a^{2} \sin ^{2} \phi+c^{2}}+a \cos \phi \log \left\{m f-a \cos \phi+\sqrt{\left.(m f-a \cos \phi)^{2}+a^{2} \sin ^{2} \phi+c^{2}\right\}}\right\}\right.
$$

to be taken from $m=0$ to $m=1$; and we thus obtain

$$
\begin{aligned}
V= & \int d \phi\left\{\sqrt{a^{2}+c^{2}+f^{2}-2 a f \cos \phi}-\sqrt{a^{2}+c^{2}}\right. \\
& \left.+a \cos \phi\left[\log \left(f-a \cos \phi+\sqrt{a^{2}+c^{2}+f^{2}-2 a f \cos \phi}\right)-\log \left(-a \cos \phi+\sqrt{a^{2}+c^{2}}\right)\right]\right\} .
\end{aligned}
$$

Writing for shortness $\sqrt{a^{2}+c^{2}+f^{2}-2 a f \cos \phi}=\Delta$, the second line of this is

$a \sin \phi\left[\log (f-a \cos \phi+\Delta)-\log \left(-a \cos \phi+\sqrt{a^{2}+c^{2}}\right)\right]$

and we thus have

$$
-\int d \phi a^{2} \sin ^{2} \phi\left\{\frac{f+\Delta}{\Delta(f-a \cos \phi+\Delta)}-\frac{1}{-a \cos \phi+\sqrt{a^{2}+c^{2}}}\right\},
$$

$$
\begin{aligned}
V=a \sin \phi\{\log ( & \left.f-a \cos \phi+\Delta)-\log \left(-a \cos \phi+\sqrt{a^{2}+c^{2}}\right)\right\} \\
& +\int d \phi\left\{\Delta-\sqrt{a^{2}+c^{2}}-\frac{a^{2} \sin ^{2} \phi(f+\Delta)}{\Delta(f-a \cos \phi+\Delta)}+\frac{a^{2} \sin ^{2} \phi}{-a \cos \phi+\sqrt{a^{2}+c^{2}}}\right\} .
\end{aligned}
$$

19. We have

$$
\frac{f+\Delta}{\Delta(f-a \cos \phi+\Delta)}=\frac{(f+\Delta)(f-a \cos \phi-\Delta)}{\Delta\left\{(f-a \cos \phi)^{2}-\Delta^{2}\right\}}
$$

the numerator of which is $f^{2}-\Delta^{2}-a \cos \phi(f+\Delta)$,

$$
\begin{aligned}
& =f^{2}+\Delta^{2}+a \cos \phi(f-a \cos \phi-\Delta)-2 a f \cos \phi+a^{2} \cos ^{2} \phi, \\
& =-c^{2}-a^{2} \sin ^{2} \phi+a \cos \phi(f-a \cos \phi-\Delta),
\end{aligned}
$$

and the denominator is $=-\Delta\left(c^{2}+a^{2} \sin ^{2} \phi\right)$. The second line of $V$ is thus $=\int d \phi\left\{\Delta-\sqrt{a^{2}+c^{2}}-\frac{a^{2} \sin ^{2} \phi}{\Delta}+\frac{a^{3} \sin ^{2} \phi \cos \phi}{\Delta} \frac{f-a \cos \phi-\Delta}{c^{2}+a^{2} \sin ^{2} \phi}+\frac{a^{2} \sin ^{2} \phi\left(\sqrt{a^{2}+c^{2}}+a \cos \phi\right)}{c^{2}+a^{2} \sin ^{2} \phi}\right\}$, $37-2$ 
which is easily reduced to

$$
\int d \phi\left\{\frac{c^{2}+f^{2}-a f \cos \phi}{\Delta}-\frac{c^{2} a \cos \phi(f-a \cos \phi)}{\left(c^{2}+a^{2} \sin ^{2} \phi\right) \Delta}-\frac{c^{2} \sqrt{a^{2}+c^{2}}}{c^{2}+a^{2} \sin ^{2} \phi}\right\}
$$

the last term of which is $=-c \tan ^{-1} \frac{\sqrt{a^{2}+c^{2}} \tan \phi}{c}$; and we thus have

$$
\begin{aligned}
V=a \sin \phi\{\log ( & \left.f-a \cos \phi+\Delta)-\log \left(-a \cos \phi+\sqrt{a^{2}+c^{2}}\right)\right\}-c \tan ^{-1} \frac{\sqrt{a^{2}+c^{2}} \tan \phi}{c} \\
& +\int d \phi\left\{\frac{c^{2}+f^{2}-a f \cos \phi}{\Delta}-\frac{c^{2} a \cos \phi(f-a \cos \phi)}{\left(c^{2}+a^{2} \sin ^{2} \phi\right) \Delta}\right\}
\end{aligned}
$$

between the limits $0,2 \pi$; or, finally,

$$
V=-2 c \pi+2 \int_{0}^{\pi} d \phi\left\{\frac{c^{2}+f^{2}-a f \cos \phi}{\Delta}-\frac{c^{2} a \cos \phi(f-a \cos \phi)}{\left(c^{2}+a^{2} \sin ^{2} \phi\right) \Delta}\right\}
$$

in partial verification whereof observe that for $a=0$ we have $\Delta=\sqrt{c^{2}+f^{2}}$, and the value becomes

$$
V=2 \pi\left(\sqrt{c^{2}+f^{2}}-c\right),
$$

which, writing therein $g$ in place of $f$, agrees with a foregoing result.

20. The process applied to finding the Potential of the Ellipse is really applicable step by step to the Circle; but if we begin by assuming $g=f$, it presents itself under a different and simplified form. Starting from

$$
V=f^{2} \int m d m \int \frac{d \phi}{\sqrt{a^{2}+c^{2}+m^{2} f^{2}-2 m a f \cos \phi}}
$$

for convenience we assume

$$
\begin{aligned}
P^{2}+Q^{2} & =a^{2}+c^{2}+m^{2} f^{2}, \\
P Q & =m a f
\end{aligned}
$$

thereby converting the radical into $\sqrt{P^{2}+Q^{2}-2 P Q \cos \phi}$. Writing also

$$
\Omega=a^{4}+c^{4}+m^{4} f^{4}+2 a^{2} c^{2}+2 m^{2} c^{2} f^{2}-2 m^{2} a^{2} f^{2}, \quad=\left(P^{2}-Q^{2}\right)^{2},
$$

and hence assuming $P^{2}-Q^{2}=\sqrt{\Omega}$, and combining with the foregoing equation

we have

$$
P^{2}+Q^{2}=a^{2}+c^{2}+m^{2} f^{2},
$$

$$
\begin{aligned}
& P^{2}=\frac{1}{2}\left(a^{2}+c^{2}+m^{2} f^{2}+\sqrt{\Omega}\right), \\
& Q^{2}=\frac{1}{2}\left(a^{2}+c^{2}+m^{2} f^{2}-\sqrt{\Omega}\right) .
\end{aligned}
$$

21. This being so, the transformation-equations to the new variable $T$ are

$$
\begin{array}{ll}
\cos \phi=\frac{P \cos T+Q}{P+Q \cos T}, \text { whence } & \cos T=\frac{P \cos \phi-Q}{P-Q \cos \phi}, \\
\sin \phi=\frac{\sqrt[4]{\Omega} \sin T}{P+Q \cos T}, & \sin T=\frac{\sqrt[4]{\Omega} \sin \phi}{P-Q \cos \phi}
\end{array}
$$


and also

We find moreover

$$
\sqrt{\Omega}=(P+Q \cos T)(P-Q \cos \phi),=P^{2}-Q^{2} .
$$

and

$$
d \phi=\frac{\sqrt[4]{\Omega} d T}{P+Q \cos T}, \quad d T=\frac{\sqrt[4]{\Omega} d \phi}{P-Q \cos \phi},
$$

whence

$$
P^{2}+Q^{2}-2 P Q \cos \phi=\frac{\sqrt{\Omega}(P-Q \cos T)}{P+Q \cos T},
$$

and hence

$$
\frac{d \phi}{\sqrt{P^{2}+Q^{2}-2 P Q \cos \phi}}=\frac{d T}{\sqrt{P^{2}-Q^{2} \cos ^{2} T}}
$$

$$
V=f^{2} \int m d m \int \frac{d T}{\sqrt{P^{2}-Q^{2} \cos ^{2} T}}
$$

where the limits of $T$ are from 0 to $2 \pi$, or, what is the same thing, we may multiply by 4 , and take them to be $0, \frac{1}{2} \pi$.

22. Assuming next

we have

$$
t=P^{2}-m^{2} f^{2}+\left(P^{2}-Q^{2}\right) \cot ^{2} T
$$

$$
\begin{aligned}
& t-P^{2}+m^{2} f^{2}=\left(P^{2}-Q^{2}\right) \frac{\cos ^{2} T}{\sin ^{2} T}, \\
& t-Q^{2}+m^{2} f^{2}=\left(P^{2}-Q^{2}\right) \frac{1}{\sin ^{2} T}, \\
& t \quad+m^{2} f^{2}=\left(P^{2}-Q^{2} \cos ^{2} T\right) \frac{1}{\sin ^{2} T}
\end{aligned}
$$

and thence

$$
\sqrt{t-P^{2}+m^{2} f^{2} \cdot t-Q^{2}+m^{2} f^{2} \cdot t+m^{2} f^{2}}=\left(P^{2}-Q^{2}\right) \frac{\cos T}{\sin ^{3} T} \sqrt{P^{2}-Q^{2} \cos ^{2} T}
$$

also

and consequently

$$
d t=-2\left(P^{2}-Q^{2}\right) \frac{\cos T}{\sin ^{3} T} d T
$$

$$
\frac{d t}{\sqrt{t-P^{2}+m^{2} f^{2} \cdot t-Q^{2}+m^{2} f^{2} \cdot t+m^{2} f^{2}}}=\frac{-2 d T}{\sqrt{P^{2}-Q^{2} \cos ^{2} T}} .
$$

$T=0$ gives $t=\infty$, and $T=\frac{1}{2} \pi$ gives $t=P^{2}-m^{2} f^{2},=G$ suppose; and we thus have

$$
V=2 f^{2} \int m d m \int_{G}^{\infty} \frac{d t}{\sqrt{t-P^{2}+m^{2} f^{2} \cdot t-Q^{2}+m^{2} f^{2} \cdot t+m^{2} f^{2}}} .
$$

23. We have

$$
\left(t-P^{2}+m^{2} f^{2}\right)\left(t-Q^{2}+m^{2} f^{2}\right)=t^{2}+\left(m^{2} f^{2}-a^{2}-c^{2}\right) t-m^{2} c^{2} f^{2},
$$

or, putting $m^{2} t$ in the place of $t$, this is

$$
=m^{2}\left\{m^{2} t^{2}+\left(m^{2} f^{2}-a^{2}-c^{2}\right) t-c^{2} f^{2}\right\},
$$


or, what is the same thing,

$$
=m^{2} t\left(t+f^{2}\right)\left\{m^{2}-\frac{a^{2}}{t+f^{2}}-\frac{c^{2}}{t}\right\}
$$

whence, completing the substitution, we have

$$
V=2 f^{2} \int m d m \int_{\theta}^{\infty} \frac{1}{\sqrt{m^{2}-\frac{a^{2}}{t+f^{2}}-\frac{c^{2}}{t}}} \frac{d t}{\sqrt{t}\left(t+f^{2}\right)},
$$

where the inferior limit $\theta,=\frac{1}{m^{2}} G,=\frac{1}{m^{2}} P^{2}-f^{2}$ is, in fact, the positive root of the equation

$$
m^{2}-\frac{a^{2}}{\theta+f^{2}}-\frac{c^{2}}{\theta}=0
$$

24. We may hence integrate in regard to $m$, through the sign $\int d t$, in the same way as if $\theta$ were constant; viz. we have

$$
V=2 f^{2}\left[\int_{\theta}^{\infty} \sqrt{m^{2}-\frac{a^{2}}{t+f^{2}}-\frac{c^{2}}{t}} \frac{d t}{\sqrt{t}\left(t+f^{2}\right)}\right]
$$

where the function of $m$ is to be taken between the limits $0,1:$ for $m=0$, we have $\theta=\infty$, and the function vanishes; hence, writing $m=1$, we obtain

$$
V=2 f^{2} \int_{\theta}^{\infty} \sqrt{1-\frac{a^{2}}{t+f^{2}}-\frac{c^{2}}{t}} \frac{d t}{\sqrt{t}\left(t+f^{2}\right)}
$$

where $\theta$ now denotes the positive root of

$$
1-\frac{a^{2}}{\theta+f^{2}}-\frac{c^{2}}{\theta}=0
$$

25. But it is interesting to reverse the transfurmation, so as to bring the radical back into its original form. For this purpose, taking now

and consequently

$$
\begin{aligned}
P^{2}+Q^{2} & =a^{2}+c^{2}+f^{2}, \\
P Q & =a f,
\end{aligned}
$$

where

$$
\begin{aligned}
& P^{2}=\frac{1}{2}\left(a^{2}+c^{2}+f^{2}+\sqrt{\Omega}\right), \\
& Q^{2}=\frac{1}{2}\left(a^{2}+c^{2}+f^{2}-\sqrt{\Omega}\right),
\end{aligned}
$$

and writing

$$
\Omega=a^{4}+c^{4}+f^{4}+2 a^{2} c^{2}+2 c^{2} f^{2}-2 a^{2} f^{2},
$$

we first obtain

$$
t=P^{2}-f^{2}+\left(P^{2}-Q^{2}\right) \cot ^{2} T,
$$

$$
V=f^{2} \int_{0}^{2 \pi} \frac{\Omega \cos ^{2} T d T}{\left(P^{2}-Q^{2} \cos ^{2} T-f^{2} \sin ^{2} T\right)\left(P^{2}-Q^{2} \cos ^{2} T\right)^{\frac{3}{2}}}
$$


and then, writing

$$
\begin{aligned}
\cos T & =\frac{P \cos \phi-Q}{P-Q \cos \phi}, \\
\sin T & =\frac{\sqrt[4]{\Omega} \sin \phi}{P-Q \cos \phi},
\end{aligned}
$$

we bring in the variable $\phi$. But it is important to remark that this is not the quantity which was, at the beginning of the investigation, represented by this letter, and that it is not easy to see the connexion between the two quantities $\phi$. We find

$$
V=f^{2} \int_{0}^{2 \pi} \frac{(P-Q \cos \phi)^{2}(P \cos \phi-Q)^{2} d \phi}{\left(a^{2}+c^{2}+f^{2} \cos ^{2} \phi-2 a f \cos \phi\right)\left(a^{2}+c^{2}+f^{2}-2 a f \cos \phi\right)^{\frac{3}{2}}} .
$$

26. To reduce this, write as before

and also

$$
\Delta=\sqrt{a^{2}+c^{2}+f^{2}-2 a f \cos \phi},
$$

$$
\Phi=a^{2}+c^{2}-2 a f \cos \phi+f^{2} \cos ^{2} \phi,
$$

so that the denominator in the integral is $=\Phi \Delta^{3}$.

We have

and hence

$$
\begin{aligned}
(P-Q \cos \phi)^{2}(P \cos \phi-Q)^{2} & =\left(\Delta^{2}-Q^{2} \sin ^{2} \phi\right)\left(\Delta^{2}-P^{2} \sin ^{2} \phi\right), \\
& =\Delta^{4}-\left(a^{2}+c^{2}+f^{2}\right) \Delta^{2} \sin ^{2} \phi+a^{2} f^{2} \sin ^{4} \phi \\
& =\Delta^{2}\left\{\Delta^{2}-\left(c^{2}+f^{2}\right) \sin ^{2} \phi\right\}-a^{2} \sin ^{2} \phi\left(\Delta^{2}-f^{2} \sin ^{2} \phi\right), \\
& =\Delta^{2}\left\{\Delta^{2}-\left(c^{2}+f^{2}\right) \sin ^{2} \phi\right\}-a^{2} \sin ^{2} \phi . \Phi
\end{aligned}
$$

$$
V=\int \frac{f^{2}\left[\Delta^{2}-\left(c^{2}+f^{2}\right) \sin ^{2} \phi\right] d \phi}{\Phi \Delta}-a^{2} f^{2} \int \frac{\sin ^{2} \phi d \phi}{\Delta^{3}},
$$

the limits being always $0,2 \pi$. But we have identically

and thence

$$
\frac{d}{d \phi} \frac{\sin \phi}{\Delta}=\frac{\cos \phi}{\Delta}-\frac{a f \sin ^{2} \phi}{\Delta^{3}}
$$

$$
\int \frac{\sin ^{2} \phi d \phi}{\Delta^{3}}=-\frac{1}{a f}\left(\frac{\sin \phi}{\Delta}\right)-\frac{1}{a f} \int \frac{\cos \phi d \phi}{\Delta},
$$

where the term $\left(\frac{\sin \phi}{\Delta}\right)$ is to be taken between the limits, but for the present I retain it as it stands. Moreover, $\Delta^{2}=\Phi+f^{2} \sin ^{2} \phi$, and consequently

$$
\Delta^{2}-\left(c^{2}+f^{2}\right) \sin ^{2} \phi=\Phi-c^{2} \sin ^{2} \phi,
$$

and we thus obtain the result

$$
V=a f\left(\frac{\sin \phi}{\Delta}\right)-a f \int \frac{\cos \phi d \phi}{\Delta}+f^{2} \int \frac{d \phi}{\Delta}-c^{2} f^{2} \int \frac{\sin ^{2} \phi d \phi}{\Phi \Delta},
$$

where the denominators under the integral signs are

$$
\Delta,=\sqrt{a^{2}+c^{2}+f^{2}-2 a f \cos \phi}, \text { and } \Phi \Delta,=\left(a^{2}+c^{2}-2 a f \cos \phi+f^{2} \cos ^{2} \phi\right) \Delta .
$$


27. We may, by a transformation such as that for the change of parameter in an elliptic integral of the third kind, make the denominators to be $\Delta$ and $\left(c^{2}+a^{2} \sin ^{2} \phi\right) \Delta$; viz. for this purpose we assume $\Lambda=\tan ^{-1} \frac{B \Delta}{A}$, where $B$ and $A$ are functions of $\phi$ such that we have identically $A^{2}+B^{2} \Delta^{2}=\left(c^{2}+a^{2} \sin ^{2} \phi\right)\left(a^{2}+c^{2}-2 a f \cos \phi+f^{2} \cos ^{2} \phi\right)$; the values of $B, A$ are found to be $c \cos \phi$ and $\sin \phi\left(a^{2}+c^{2}-a f \cos \phi\right)$, whence, dividing each of these for greater convenience by $\sin \phi$, we have

$$
\Lambda=\tan ^{-1}\left(\frac{c \cot \phi \Delta}{a^{2}+c^{2}-a f \cos \phi}\right),
$$

so that, writing now $B, A=c \cot \phi$ and $a^{2}+c^{2}-a f \cos \phi$ respectively, the value is

where

$$
\Lambda=\tan ^{-1}\left(\frac{B \Delta}{A}\right)
$$

$$
A^{2}+B^{2} \Delta^{2}=\frac{1}{\sin ^{2} \phi} \Pi \Phi
$$

and, as before, $\Phi=a^{2}+c^{2}-2 a f \cos \phi+f^{2} \cos ^{2} \phi$, and also $\Pi=c^{2}+a^{2} \sin ^{2} \phi$. We have

and then

$$
\frac{d \Lambda}{d \phi}=\frac{\left(A B^{\prime}-A^{\prime} B\right) \Delta^{2}+\frac{1}{2} A B\left(\Delta^{2}\right)^{\prime}}{\left(A^{2}+B^{2} \Delta^{2}\right) \Delta}, \quad\left(A^{\prime}=\frac{d A}{d \phi}, \& c .\right) ;
$$

$$
\begin{aligned}
& A B^{\prime}-A^{\prime} B=\frac{a}{\sin ^{2} \phi}\left(-a^{2}-c^{2}+a f \cos ^{3} \phi\right), \\
& \frac{1}{2} A B\left(\Delta^{2}\right)^{\prime}=\frac{c \sin \phi \cos \phi}{\sin ^{2} \phi}\left(a^{2}+c^{2}-a f \cos \phi\right) a f \sin \phi,
\end{aligned}
$$

and the numerator thus is

$$
\begin{aligned}
& \frac{c}{\sin ^{2} \phi}\left\{\left(-a^{2}-c^{2}+a f \cos ^{3} \phi\right)\right.\left(a^{2}+c^{2}+f^{2}-2 a f \cos \phi\right) \\
&\left.+a f \cos \phi\left(1-\cos ^{2} \phi\right)\left(a^{2}+c^{2}-a f \cos \phi\right)\right\},
\end{aligned}
$$

which is in fact

$$
\begin{aligned}
& =\frac{c}{\sin ^{2} \phi}\left\{-\left(c^{2}+a^{2} \sin ^{2} \phi\right)\left(a^{2}+c^{2}+f^{2}-2 a f \cos \phi\right)\right. \\
& \left.\quad+\left(a f \cos \phi-a^{2} \cos ^{2} \phi\right)\left(a^{2}+c^{2}-2 a f \cos \phi+f^{2} \cos ^{2} \phi\right)\right\}, \\
& =\frac{c}{\sin ^{2} \phi}\left\{-\Pi \Delta^{2}+\left(a f \cos \phi-a^{2} \cos ^{2} \phi\right) \Phi\right\} ;
\end{aligned}
$$

or, what is the same thing,

$$
=\frac{c}{\sin ^{2} \phi}\left\{-\Pi \phi-\Pi f^{2} \sin ^{2} \phi+\left(a f \cos \phi-a^{2} \cos ^{2} \phi\right) \Phi\right\},
$$

and the denominator, by what precedes, is

$$
=\frac{1}{\sin ^{2} \phi} \cdot \Pi \Phi \Delta .
$$


We thus have

$$
\frac{1}{c} \frac{d \Lambda}{d \phi}=-\frac{1}{\Delta}-\frac{f^{2} \sin ^{2} \phi}{\Phi \Delta}+\frac{a f \cos \phi-a^{2} \cos ^{2} \phi}{\Pi \Delta},
$$

whence, by integration,

$$
\frac{1}{c} \tan ^{-1}\left(\frac{c \cot \phi \Delta}{a^{2}+c^{2}-a f \cos \phi}\right)=-\int \frac{d \phi}{\Delta}+\int \frac{\left(a f \cos \phi-a^{2} \cos ^{2} \phi\right) d \phi}{\Pi \Delta}-f^{2} \int \frac{\sin ^{2} \phi d \phi}{\Phi \Delta},
$$

which is the required formula of transformation.

28. Multiplying by $c^{2}$, and subtracting from the value of $V$, we find

$$
\begin{aligned}
V=c \tan ^{-1}\left(\frac{c \cot \phi \Delta}{a^{2}+c^{2}-a f \cos \phi}\right)+a f\left(\frac{\sin \phi}{\Delta}\right) \\
+\int \frac{\left(c^{2}+f^{2}-a f \cos \phi\right) d \phi}{\Delta}-c^{2} a \int \frac{\cos \phi(f-a \cos \phi) d \phi}{\left(c^{2}+a^{2} \sin ^{2} \phi\right) \Delta},
\end{aligned}
$$

which is to be taken between the limits 0 and $2 \pi$; viz. we thus have

$$
V=-2 c \pi+2 \int_{0}^{\pi} \frac{\left(c^{2}+f^{2}-a f \cos \phi\right) d \phi}{\Delta}-2 c^{2} a \int_{0}^{\pi} \frac{\cos \phi(f-a \cos \phi) d \phi}{\left(c^{2}+a^{2} \sin ^{2} \phi\right) \Delta},
$$

agreeing with a former result.

29. But this former result, previous to the final step of taking the integrals between the limits, was

$$
\begin{array}{r}
V=2 a \sin \phi \log \left(\frac{f-a \cos \phi+\Delta}{-a \cos \phi+\sqrt{a^{2}+c^{2}}}\right)-c \tan ^{-1}\left(\frac{\sqrt{a^{2}+c^{2}} \tan \phi}{c}\right) \\
+\int \frac{\left(c^{2}+f^{2}-a f \cos \phi\right) d \phi}{\Delta}-c^{2} a \int \frac{\cos \phi(f-a \cos \phi) d \phi}{\left(c^{2}+a^{2} \sin ^{2} \phi\right) \Delta}
\end{array}
$$

viz. the integrals are the same, but the integrated terms are altogether different; the explanation of course is that the $\phi$ 's are different in the two formulæ, which therefore do not correspond element by element but only in their ultimate value between the limits.

30. In order to discuss numerically the Potential of the Circle,

$$
V=2 f^{2} \int_{\theta}^{\infty} \frac{\sqrt{\left(t-\theta \cdot t+\frac{c^{2} f^{2}}{\theta}\right)} d t}{t\left(t+f^{2}\right) \sqrt{t+f^{2}}}
$$

this must be reduced to elliptic functions. Writing $t=\theta+x^{2}$, we have

$$
V=4 f^{2} \int_{0}^{\infty} \frac{x^{2} \sqrt{x^{2}+\beta^{2}} d x}{\left(x^{2}+\theta\right)\left(x^{2}+\alpha^{2}\right)^{\frac{3}{2}}}
$$

C. IX. 
if for shortness

$$
\begin{aligned}
& \theta+f^{2}=\alpha^{2} \\
& \theta+\frac{c^{2} f^{2}}{\theta}=\beta^{2}
\end{aligned}
$$

The constants $\alpha, \beta, \theta$ may be considered as replacing the original constants $a, c, f$; viz. from the last two equations and the equation

we deduce

$$
\frac{a^{2}}{\theta+f^{2}}+\frac{c^{2}}{\theta}=1
$$

$$
a^{2}=\frac{\alpha^{2}\left(\alpha^{2}-\beta^{2}\right)}{\alpha^{2}-\theta}, \quad c^{2}=\frac{\theta\left(\beta^{2}-\theta\right)}{\alpha^{2}-\theta}, \quad f^{2}=\alpha^{2}-\theta
$$

showing that $\alpha^{2}, \beta^{2}, \theta$ are in order of decreasing magnitude; viz. $\alpha^{2}-\beta^{2}, \beta^{2}-\theta, \alpha^{2}-\theta$ are all positive. The formula may be written

$$
\frac{1}{4} V=\left(\alpha^{2}-\theta\right) \int_{0}^{\infty} \frac{x^{2}\left(x^{2}+\beta^{2}\right) d x}{\left(x^{2}+\theta\right)\left(x^{2}+\alpha^{2}\right) \sqrt{x^{2}+\alpha^{2} \cdot x^{2}+\beta^{2}}}
$$

which, in virtue of the identity

$$
\left(\alpha^{2}-\theta\right) x^{2}\left(x^{2}+\beta^{2}\right)=\left(\alpha^{2}-\theta\right)\left(x^{2}+\theta\right)\left(x^{2}+\alpha^{2}\right)-\alpha^{2}\left(\alpha^{2}-\beta^{2}\right)\left(x^{2}+\theta\right)-\theta\left(\beta^{2}-\theta\right)\left(x^{2}+\alpha^{2}\right),
$$

becomes

$$
\begin{aligned}
\frac{1}{4} V= & \left(\alpha^{2}-\theta\right) \int_{0}^{\infty} \frac{d x}{\sqrt{x^{2}+\alpha^{2} \cdot x^{2}+\beta^{2}}} \\
& -\alpha^{2}\left(\alpha^{2}-\beta^{2}\right) \int_{0}^{\infty} \frac{d x}{\left(x^{2}+\alpha^{2}\right) \sqrt{x^{2}+\alpha^{2} \cdot x^{2}+\beta^{2}}} \\
& -\theta\left(\beta^{2}-\theta\right) \int_{0}^{\infty} \frac{d x}{\left(x^{2}+\theta\right) \sqrt{x^{2}+\alpha^{2} \cdot x^{2}+\beta^{2}}}
\end{aligned}
$$

31. Writing here $x=\alpha \cot u$, and therefore $d x=-\alpha \operatorname{cosec}^{2} u d u$, to the values $x=\infty, 0$ correspond $u=0, \frac{1}{2} \pi$, and we have

$$
\begin{gathered}
\frac{1}{4} V=\int_{0}^{\frac{1}{2} \pi} \frac{d u}{\sqrt{\alpha^{2} \cos ^{2} u+\beta^{2} \sin ^{2} u}}\left\{\alpha^{2}-\theta-\left(\alpha^{2}-\beta^{2}\right) \sin ^{2} u-\frac{\theta\left(\beta^{2}-\theta\right) \sin ^{2} u}{\alpha^{2} \cos ^{2} u+\theta \sin ^{2} u}\right\} \\
=\int_{0}^{\frac{2}{2} \pi} \frac{d u}{\sqrt{\alpha^{2} \cos ^{2} u+\beta^{2} \sin ^{2} u}}\left\{\alpha^{2}-\theta+\frac{\theta\left(\beta^{2}-\theta\right)}{\alpha^{2}-\theta}-\left(\alpha^{2}-\beta^{2}\right) \sin ^{2} u-\frac{\alpha^{2} \theta\left(\beta^{2}-\theta\right)}{\alpha^{2}-\theta} \frac{1}{\alpha^{2} \cos ^{2} u+\theta \sin ^{2} u}\right\} .
\end{gathered}
$$

Writing $k^{2}=1-\frac{\beta^{2}}{\alpha^{2}}$, we have

and thence

$$
\sqrt{\alpha^{2} \cos ^{2} u+\beta^{2} \sin ^{2} u}=\alpha \sqrt{1-k^{2} \sin ^{2} u},
$$

$$
\frac{1}{4} V=\int_{0}^{\frac{1}{2} \pi} \frac{d u}{\sqrt{1-k^{2} \sin ^{2} u}} \times\left\{\alpha\left(1-k^{2} \sin ^{2} u\right)-\frac{k^{2} \theta}{1-\frac{\theta}{\alpha^{2}}}-\frac{\frac{\theta}{\alpha} \frac{\beta^{2}-\theta}{\alpha^{2}}}{1-\frac{\theta}{\alpha^{2}}} \frac{1}{1-\left(1-\frac{\theta}{\alpha^{2}}\right) \sin ^{2} u}\right\}
$$


viz. writing $n=-1+\frac{\theta}{\alpha^{2}}$ (so that $n$ is negative and in absolute magnitude $<1$ ), and moreover $\beta^{2}=\alpha^{2} k^{\prime 2}$ and $\theta=(n+1) \alpha^{2}$, this is

$$
\frac{1}{4} V=\int_{0}^{\frac{1}{2} \pi} \frac{d u}{\sqrt{1-k^{2} \sin ^{2} u}} \times\left\{\left(1-k^{2} \sin ^{2} u\right) \alpha+\frac{k^{2}}{n}(n+1) \alpha-\frac{n+1 \cdot n+k^{2}}{n} \alpha \frac{1}{1+n \sin ^{2} u}\right\} ;
$$

viz. this is

$$
=\alpha\left\{E_{1} k+k^{2} \frac{n+1}{n} F_{1} k-\frac{n+1 \cdot n+k^{2}}{n} \Pi_{1}(n, k)\right\} .
$$

32. This may be further reduced by substituting for the complete function $\Pi_{1}(n, k)$, its value; viz. writing

$$
n=\left(-1+\frac{\theta}{\alpha^{2}}\right)=-1+k^{\prime 2} \sin ^{2} \lambda,
$$

that is, $\sin ^{2} \lambda=\frac{\theta}{\beta^{2}}$; then, writing the value first in the form

and observing that

$$
\alpha\left\{E_{1} k-(n+1) F_{1} k-\frac{n+1 \cdot n+k^{2}}{n}\left[\Pi_{1}(n, k)-F_{1} k\right]\right\},
$$

$$
\begin{aligned}
\frac{n+1 \cdot n+k^{2}}{n}\left[\Pi_{1}(n, k)-F_{1} k\right] & =\frac{k^{\prime 4} \sin ^{2} \lambda \cos ^{2} \lambda}{1-k^{\prime 2} \sin ^{2} \lambda}\left[\Pi_{1}(n, k)-F_{1} k\right] \\
& =\frac{k^{\prime 2} \sin \lambda \cos \lambda}{\sqrt{1-k^{\prime 2} \sin ^{2} \lambda}}\left\{\frac{1}{2} \pi+\left(F_{1} k-E_{1} k\right) F\left(k^{\prime}, \lambda\right)-F_{1} k \cdot E\left(k^{\prime}, \lambda\right)\right\},
\end{aligned}
$$

we have

$$
\frac{1}{4} V=\alpha\left\{E_{1} k-k^{\prime 2} \sin ^{2} \lambda F_{1} k-\frac{k^{\prime 2} \sin \lambda \cos \lambda}{\sqrt{1-k^{\prime 2} \sin ^{2} \lambda}}\left[\frac{1}{2} \pi+\left(F_{1} k-E_{1} k\right) F\left(k^{\prime}, \lambda\right)-F_{1}^{\prime} k . E\left(k^{\prime}, \lambda\right)\right]\right\},
$$

where

$$
\alpha^{2}=\theta+f^{2}, \quad k^{2}=1-\frac{\beta^{2}}{\alpha^{2}}, \quad=1-\frac{\theta+\frac{c^{2} f^{2}}{\theta}}{\theta+f^{2}},=\frac{f^{2}\left(1-\frac{c^{2}}{\theta}\right)}{\theta+f^{2}},
$$

or, what is the same thing,

$$
k=\frac{a f}{f^{2}+\theta}, \quad \sin ^{2} \lambda=\frac{\theta}{\beta^{2}}, \quad=\frac{1}{1+\frac{c^{2} f^{2}}{\theta^{2}}},
$$

$\theta$ being, it will be recollected, the positive root of

$$
\frac{a^{2}}{f^{2}+\theta}+\frac{c^{2}}{\theta}=1
$$

33. Thus when in particular $a=0$, we have $\theta=c^{2}$, and thence

$$
\alpha=\sqrt{c^{2}+f^{2}}, \quad k=0, \quad k^{\prime}=1, \quad \sin \lambda=\frac{c}{\sqrt{c^{2}+f^{2}}} ;
$$


whence

or

$$
\begin{aligned}
\frac{1}{4} V & =\frac{1}{2} \pi \sqrt{c^{2}+f^{2}}\left\{1-\sin ^{2} \lambda-\sin \lambda(1-\sin \lambda)\right\}, \\
& =\frac{1}{2} \pi \sqrt{c^{2}+f^{2}}(1-\sin \lambda), \quad=\frac{1}{2} \pi\left(\sqrt{c^{2}+f^{2}}-c\right),
\end{aligned}
$$

which is right.

$$
V=2 \pi\left(\sqrt{c^{2}+f^{2}}-c\right)
$$

34. If $c=0, a$ being $>f$, then $\theta=a^{2}-f^{2}, k=\frac{f}{a}, \lambda=\frac{1}{2} \pi, \alpha=a$; so that, retaining $k$ as standing for its value $\frac{f}{a}$, we have

$$
\frac{1}{4} V=a\left(E_{1} k-k^{\prime 2} F_{1} k\right), \text { or } V=4 a\left(E_{1} k-k^{\prime 2} F_{1} k\right),
$$

which may easily be verified.

If $c=0, a$ being $<f$, then, recurring to the original equation for the determination of $\theta$, viz. $\left(\theta+f^{2}\right)^{2} \theta\left(\frac{a^{2}}{\theta+f^{2}}+\frac{c^{2}}{\theta}-1\right)=0$, which for $c=0$ becomes $\theta\left(\theta+f^{2}\right)\left(\theta-a^{2}+f^{2}\right)=0$, we have (as the positive root of this equation) $\theta=0$; whence $\alpha=f$; also, observing that $1-\frac{c^{2}}{\theta}=\frac{a^{2}}{f^{2}}, k=\frac{a}{f}$, and $\sin ^{2} \lambda=\frac{\theta}{\theta+\frac{c^{2}}{\theta} f^{2}}\left(\right.$ where $\frac{c^{2}}{\theta}$ is finite $),=0$, and retaining $k$ to denote its value $=\frac{a}{f}$, we obtain $\frac{1}{4} V=f E_{1} k$, or $V=4 f E_{1} k$.

If $a=f$, then in each of the formulæ $k=1$; and since in the first formula $k^{\prime 2} F_{1} k, k$ nearly $=1$, is $=k^{\prime 2} \log \frac{4}{k^{\prime}}$, vanishing for $k=1$ or $k^{\prime}=0$, we have $V=4 f E_{1} 1,=4 f$.

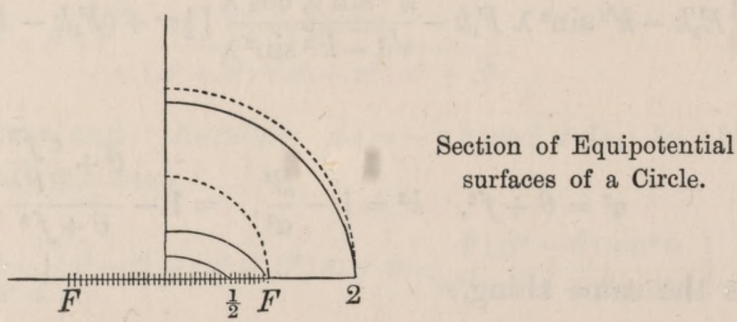

It would be interesting to consider the value of the potential at different points of the ellipse $\frac{a^{2}}{f^{2}+\theta}+\frac{c^{2}}{\theta}=1$ ( $\theta$ constant, $a, c$ current coordinates). For this purpose writing $a=\sqrt{f^{2}+\theta} \cos q, c=\sqrt{\theta} \sin q$, we should have $\alpha=\sqrt{f^{2}+\theta}$ (a constant), and

$$
\begin{gathered}
k=\frac{f \cos q}{\sqrt{f^{2}+\theta}}, \quad k^{\prime}=\frac{\sqrt{\theta+f^{2} \sin ^{2} q}}{\sqrt{f^{2}+\theta}}, \\
\sin \lambda=\frac{\sqrt{\theta}}{\sqrt{\theta+f^{2} \sin ^{2} q}}, \quad \cos \lambda=\frac{f \sin q}{\sqrt{\theta+f^{2} \sin ^{2} q}} ;
\end{gathered}
$$

and then $V$ through $k, k^{\prime}, \lambda$, is a given function of $q$. 
35. Suppose, to fix the ideas, $f=1$, and consider the points $(0, c)$ and $(a, 0)$, which have equal potentials. First, if $a>f$ (that is, $a>1$ ), then writing $k=\frac{1}{a}$, the relation is

and we have

$$
2 \pi\left(\sqrt{1+c^{2}}-c\right)=\frac{4}{k}\left(E_{1} k-k^{\prime 2} F_{1} k\right)
$$

$$
F_{1} 30^{\circ}=1 \cdot 68575, \quad E_{1} 30^{\circ}=1 \cdot 46746, \quad \frac{4}{\pi}=1 \cdot 27324 .
$$

Secondly, if $a<f$ (that is, $a<1$ ), then writing $k=a$, the relation is

$$
2 \pi\left(\sqrt{1+c^{2}}-c\right)=4 E_{1} k .
$$

(1) In particular $a=\frac{1}{2},=\sin 30^{\circ}$, this is

$$
\sqrt{1+c^{2}}-c=\frac{2}{\pi} E_{1} 30^{\circ} \quad=.93421 .
$$

(2) $a=1$, then

$$
\sqrt{1+c^{2}}-c=\frac{2}{\pi} \quad=\cdot 63662
$$

(3) $a=2, k=\frac{1}{2},=\sin 30^{\circ}$,

$$
\sqrt{1+c^{2}}-c=\frac{4}{\pi}\left\{E_{1}\left(30^{\circ}\right)-\frac{3}{4} F_{1}\left(30^{\circ}\right)\right\}=\cdot 25866 .
$$

But if $\sqrt{1+c^{2}}-c=m$, then $c=\frac{1}{2}\left(\frac{1}{m}-m\right)$; whence

\begin{tabular}{l|c}
$a$ & $c$ \\
\hline 0 & \multicolumn{1}{c}{0} \\
$\frac{1}{2}$ & $\cdot 06810$ \\
1 & $\cdot 46709$ \\
2 & $1 \cdot 80376$
\end{tabular}

for the values of $c$, corresponding to the foregoing values of $a$. 